Article

\title{
Cross-Coupled Contouring Control of Multi-DOF Robotic Manipulator
}

\author{
Puren Ouyang ${ }^{1,2, *}$, Yuqi Hu ${ }^{2}$, Wenhui Yue ${ }^{2}$ and Deshun Liu ${ }^{2}$ \\ 1 Department of Aerospace Engineering, Ryerson University, Toronto, ON M5B 2K3, Canada \\ 2 College of Mechanical and Electrical Engineering, Hunan University of Science and Technology, \\ Xiangtan 411201, China; huyuqi2003@126.com (Y.H.); whyue@hnust.edu.cn (W.Y.); \\ liudeshun@hnust.edu.cn (D.L.) \\ * Correspondence: pouyang@ryerson.ca; Tel.: +1-416-979-5000 (ext. 4928)
}

Academic Editor: Javier Del Ser Lorente

Received: 28 August 2016; Accepted: 18 November 2016; Published: 24 November 2016

\begin{abstract}
Reduction of contour error is a very important issue for high precise contour tracking applications, and many control systems were proposed to deal with contour tracking problems for two/three axial translational motion systems. However, there is no research on cross-coupled contour tracking control for serial multi-DOF robot manipulators. In this paper, the contouring control of multi-DOF serial manipulators is developed for the first time and a new cross-coupled PD (CC-PD) control law is proposed, based on contour errors of the end-effector and tracking errors of the joints. It is a combination of PD control for trajectory tracking at joint level and PD control for contour tracking at the end-effector level. The contour error of the end-effector is transformed to the equivalent tracking errors of the joints using the Jacobian regulation, and the CC-PD control law is implemented in the joint level. Stability analysis of the proposed CC-PD control system is conducted using the Lyapunov method, followed by some simulation studies for linear and nonlinear contour tracking to verify the effectiveness of the proposed CC-PD control system.
\end{abstract}

Keywords: contour tracking; contour error; contouring control; robot; cross-coupled control; PD control; Jacobian; stability analysis

\section{Introduction}

Robotic manipulators are important for automation and industrial applications in contour tracking and trajectory tracking. Contour tracking is very popular in many industrial applications such as welding, laser cutting, and machining [1-4], and contouring control is one of the most important tasks for robotics and computer numerical control machine tools [5-9]. One of the most important issues in machining is the reduction of contour errors to ensure the quality of machined parts. In robotic applications such as precision machining, a desired task is often defined in terms of the end-effector (task space) motion: contour tracking motion [10,11].

Robotic technology can provide an excellent base to reduce the contour error because of its flexibility and affordability. A contour error is defined as the shortest distance between the current position and the desired contour curve, and contour error is a key factor used to evaluate the quality of a machined part. Conventional approach to deal with such a contouring problem is to treat it as a tracking control problem for each individual axis or joint. Unfortunately, reducing tracking error does not ensure reducing the contour error, because the contour error is a result of combined tracking errors of all axes or joints, and the dynamics of each axis is coupled with other axes for a nonlinear robotic system.

To deal with contour tracking problems, the cross coupling control (CCC) method was first developed by Koren [1] for translational motion systems, followed by a variable-gain CCC in [2]. A PI 
type cross coupling controller was developed for contour tracking of a linear bi-axis motion system based on linear control theory in [3]. A position loop-based cross-coupled control was proposed for a two-axis motion stage in [4]. Cheng [5] applied the fuzzy logic technology to deal with a free-form contour following problem by varying the feed rate of the system. Some surveys about the tracking and contour error control for CNC systems were presented in [6,7].

For simple contours, the contour error can be calculated analytically as discussed in [1,2]. To calculate contour errors of complex freeform contours, the concept of equivalent error was introduced for contouring control in [12]. A polar coordinate representation method was proposed in [13] to calculate contour errors for a biaxial translational motion system. Contour error estimation procedures for three-dimensional translational machining were developed in [8,9], based on the linear dynamic model of a CNC machine system. An orthogonal global task coordinate frame [14] for the calculation of contour errors was proposed and applied to a biaxial system. It should be noted that the majority of contouring control is focusing on biaxial or multi-axial translational motion systems that are linear dynamic systems.

There are two types of applications for robot manipulators in the task space level: the task space trajectory tracking control and task space contour tracking control. For task space trajectory tracking control, Cheah [10] developed a set of task space PD control laws based on the Jacobian regulation. An adaptive tracking control was proposed for robots with unknown kinematic and dynamic properties in [11]. In addition, the adaptive technology was applied to the contour coupling control for a CNC system in [15]. As for the contouring control, a cross-coupled control algorithm based on the Lyapunov stability criteria and the recursive updating technique was proposed and applied to a two-link direct drive robot [16]. In addition, a contour control strategy based on the coordinate transformation was developed in [17].

The contour tracking problem for 2-DOF robots was discussed in [16-18], but there is no research on cross-coupled contour tracking control for multi-DOF robot manipulators. The main motivation of this research is to develop a contouring control system, called cross coupled PD (CC-PD) control, for multi-DOF robot manipulators with the combination of joint error and contour error as the feedback signals [19]. In the developed CC-PD control system, the contour errors in the end-effector are transformed to the equivalent tracking errors in the joint level through the Jacobian regulation [10], and the control law is implemented in the joint level to simplify the control system design and its implementation.

\section{Dynamic Model and New Cross-Coupled Control Law}

In this section, the dynamic model of a multi-DOF robotic manipulator is presented, followed by an introduction of the contour error and its calculation. Then a transformation method is presented to transfer the contour error at the task level to the tracking error in the joint level. After that, the CC-PD control law is proposed for contouring tracking.

\subsection{Dynamic Model of Robotic Manipulator}

The dynamic model of an n-DOF serial planar robotic manipulator with revolute joints can be expressed as [20]:

$$
M(q) \ddot{q}(t)+C(q, \dot{q}) \dot{q}(t)+G(q)+F(\dot{q})=\tau(t)
$$

where $q(t), \dot{q}(t)$, and $\ddot{q}(t)$ are $n \times 1$ joint position, velocity and acceleration vectors, defined as functions of time; $M(q)$ is the symmetric positive-definite $n \times n$ inertia matrix; $C(q, \dot{q})$ is an $n \times n$ matrix of the Coriolis and centrifugal forces; $G(q)$ is an $n \times 1$ vector of gravity terms; $F(\dot{q})$ is an $n \times 1$ vector of friction forces, and $\tau(t)$ is an $n \times 1$ vector of joint torques/forces.

In this paper, some properties of a rigid nonlinear robotic manipulator [20] associated with Equation (1) is used in the stability analysis and are described as follows.

(P1) The inertia matrix $M(q)$ is symmetric positive definite. 
(P2) The matrix $\frac{1}{2} \dot{M}(q)-C(q, \dot{q})$ is a skew symmetric matrix, i.e.,

$$
\left\{\begin{array}{l}
x^{T}(\dot{M}(q)-2 C(q, \dot{q})) x=0 \\
\dot{M}(q)=C(q, \dot{q})+C^{T}(q, \dot{q})
\end{array}\right.
$$

(P3) The matrix $C(q, \dot{q})$ satisfies

$$
\left\{\begin{array}{l}
C(q, x) y=C(q, y) x \\
C(q, x+z) y=C(q, x) y+C(q, z) y
\end{array}\right.
$$

where $x, y$, and $z$ are velocity vectors.

(P4) $M(q), C\left(q, \dot{q}_{d}\right), G(q)$, and $F(\dot{q})$ are bounded.

\subsection{Contour Error of End-Effector}

To obtain accurate contour trajectories is a fundamental requirement for modern high precision manufacturing. The contour error can be used to evaluate the quality of contour tracking, and it is defined as an orthogonal component of the derivation of an actual contour from the desired one. Figure 1 shows the relationship and difference between a tracking error and a contour error for an $X Y$ planar contour.

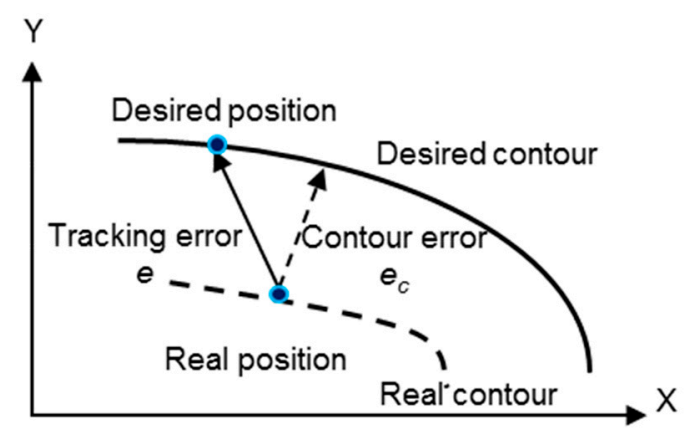

Figure 1. Tracking and contour errors for a planar contour.

Assume small joint errors are obtained during the contour tracking process, tracking errors in the joint level and at the end-effector of an n-DOF serial planar robot can be calculated as follows:

$$
\begin{aligned}
& e_{q i}(t)=q_{i d}(t)-q_{i}(t) \\
& e_{x}(t)=-\sum_{i=1}^{n} l_{i} \sum_{j=1}^{i} e_{q j}(t) \sin \left(\sum_{j=1}^{i} q_{j}(t)\right) \\
& e_{y}(t)=\sum_{i=1}^{n} l_{i} \sum_{j=1}^{i} e_{q j}(t) \cos \left(\sum_{j=1}^{i} q_{j}(t)\right)
\end{aligned}
$$

where $q_{i d}(t)$ and $l_{i}$ are the desired joint position and the length of link $i$, respectively.

For the purpose of online calculation of the contour error $e_{\mathcal{c}}$, the following simple formulae presented in [1,2] is used in this paper. For a straight-line contour, the contour error $e_{c}$ can be expressed as:

$$
e_{c}=-C_{x} e_{x}+C_{y} e_{y} \text { with } C_{x}=\sin \theta, \quad C_{y}=\cos \theta
$$

where $\theta$ is the angle between the desired straight line and the $X$-axis, $e_{x}$ and $e_{y}$ are the tracking errors of the end effector in the $X$ and $Y$ axes, respectively. It can be seen that the contour error coefficients $C_{x}$ and $C_{y}$ are constant for linear contour tracking. 
On the other hand, for a circular contour, the contour error coefficients are not constant and can be obtained by the following Equation [2]:

$$
e_{c}=-C_{x} e_{x}+C_{y} e_{y} \text { with } C_{x}=\sin \theta-\frac{e_{x}}{2 R}, \quad C_{y}=\cos \theta+\frac{e_{y}}{2 R}
$$

It should be mentioned that the angle $\theta$ is not constant but a variable for a circular contour, related to the location of the desired circle contour.

\subsection{Equivalent Tracking Error of Contour Error}

As mentioned in the introduction section, the proposed contouring control law is implemented in the joint level. Therefore, the contour error of the end-effector must be mapped to the joint tracking error. A correlation between the joint space and the task space can be easily obtained by the following forward kinematics of the robotic manipulator:

$$
P_{t}=f(q)
$$

where $f(q)$ is a nonlinear mapping function from joint space to task space through the forward kinematics of the robotic manipulator. By differentiating Equation (5), the end-effector velocity is related with the joint space velocity as:

$$
\dot{P}_{t}=\dot{f}(q)=J(q) \dot{q}
$$

where $J(q)$ is the Jacobian matrix that transforms the vector of the generalized joint velocities to the vector of the generalized end-effector velocities.

As pointed out in [10], the Jacobian transpose regulator and inverse Jacobian regulator are very robust with respect to uncertainty in kinematic parameters. Transforming of control space is necessary as the contour performance is measured in the task space but the driving actuators are controlled in the joint space. Similar to the process discussed in [10], the following equation is used to build the relationship of the errors between the task space and the joint space:

$$
\left\{\begin{array}{l}
e_{P}=J(q) e_{q} \\
\dot{e}_{P}=J(q) \dot{e}_{q}+j(q) e_{q}
\end{array}\right.
$$

where $e_{P}=\left[\begin{array}{lll}e_{x} & e_{y} & e_{\phi}\end{array}\right]^{T}$ is the error vector in the task space and $e_{q}=\left[\begin{array}{lll}e_{q_{1}} & \cdots & e_{q_{n}}\end{array}\right]^{T}$ is the error vector in the joint space, respectively.

As $e_{\phi}$ does not affect the contour error according to Equation (3), submitting Equation (7) into Equation (3), one can see that the contour error can be expressed as a function of the tracking errors in the joint space as follows:

$$
\left\{\begin{array}{l}
e_{c}=\left[\begin{array}{lll}
-C_{x} & C_{y} & 0
\end{array}\right] e_{P}=\left[\begin{array}{lll}
-C_{x} & C_{y} & 0
\end{array}\right] J(q) e_{q} \\
\dot{e}_{c}=\left[\begin{array}{lll}
-C_{x} & C_{y} & 0
\end{array}\right] \dot{e}_{P}=\left[\begin{array}{lll}
-C_{x} & C_{y} & 0
\end{array}\right]\left(j(q) e_{q}+J(q) \dot{e}_{q}\right)
\end{array}\right.
$$

\subsection{Cross-Coupled Contouring Controller}

As mentioned before, PD control is very popular for robots in industrial applications. In this paper, a cross-coupled contouring controller called CC-PD is proposed as follows:

$$
\tau=K_{p} e_{q}+K_{d} \dot{e}_{q}+K_{c p} e_{c}+K_{c d} \dot{e}_{c}
$$


where $K_{p}$ and $K_{d}$ are the proportional and derivative control gain matrices in the joint space; all these matrices are assumed to be positive definite. $K_{c p}$ and $K_{c d}$ are the proportional and derivative control vectors with positive values in the task space, respectively.

From Equation (9), one can see that the proposed CC-PD is a combination of PD control in the joint space based on the tracking errors and PD control in the task space based on the contour errors. The PD controller in the joint space is used to maintain the stability of the robotic system, while the PD controller in the task space is used to reduce the contour errors. The goal for developing such a combined controller is to improve tracking performances at the joint level and the contour performances at the task level as well.

Based on the Jacobian regulation, applying Equation (8) into Equation (9), the proposed CC-PD control law can be simplified as:

$$
\begin{gathered}
\tau=\left(K_{p}+\bar{K}_{c p}\right) e_{q}+\left(K_{d}+\bar{K}_{c d}\right) \dot{e}_{q} \\
\text { with }\left\{\begin{array}{l}
\bar{K}_{c p}=K_{c p}\left[\begin{array}{lll}
-C_{x} & C_{y} & 0
\end{array}\right] J(q)+K_{c d}\left[\begin{array}{lll}
-C_{x} & C_{y} & 0
\end{array}\right] j(q) \\
\bar{K}_{c d}=K_{c d}\left[\begin{array}{lll}
-C_{x} & C_{y} & 0
\end{array}\right] J(q)
\end{array}\right.
\end{gathered}
$$

According to Equations (10) and (11), it can be seen that the proposed CC-PD control law is an equivalent nonlinear PD control in the joint level, as the Jacobian matrix is a nonlinear function of the joint positions.

\section{Stability Analysis}

The following notations are introduced to facilitate the stability analysis. $\lambda_{m}(A)$ and $\lambda_{M}(A)$ represent the smallest and the largest eigenvalues of a positive define matrix $A$. If a square matrix $A$ is positive definite, then it is denoted as $A \succ 0$; If a square matrix $A-B$ is positive definite, then it is denoted as $A-B \succ 0$.

For positive definite matrices, the following properties [21] will be used in this paper:

(P5) If $A \succ 0$, then $A^{-1} \succ 0$; If $A \geq B \succ 0$, then $B^{-1} \geq A^{-1} \succ 0$.

(P6) If $A \succ 0$ and $B \succ 0$, then $A+B \succ 0, A B A \succ 0$, and $B A B \succ 0$.

According to P2, we have:

$$
\begin{aligned}
C(q, \dot{q}) \dot{q}_{d} & =C\left(q, \dot{q}_{d}\right) \dot{q}=C\left(q, \dot{q}_{d}\right) \dot{q}_{d}-C\left(q, \dot{q}_{d}\right) \dot{e}_{q} \\
& =C_{0} \dot{q}_{d}-C_{0} \dot{e}_{q}
\end{aligned}
$$

The dynamic model in Equation (1) can be rewritten as a function of the tracking errors in the following form.

$$
M(q) \ddot{e}_{q}+C(q, \dot{q}) \dot{e}_{q}+\left(K_{d}+\bar{K}_{c d}\right) \dot{e}_{q}+\left(K_{p}+\bar{K}_{c p}\right) e_{q}=M(q) \ddot{q}_{d}+C(q, \dot{q}) \dot{q}_{d}+G(q)+F(\dot{q})
$$

By applying Equation (12), Equation (13) is represented as the following simple form.

$$
M \ddot{e}_{q}+C \dot{e}_{q}+C_{0} \dot{e}_{q}+K_{d d} \dot{e}_{q}+K_{p p} e_{q}=\rho
$$

$$
\text { with }\left\{\begin{array}{l}
K_{p p}=K_{p}+\bar{K}_{c p} \\
K_{d d}=K_{d}+\bar{K}_{c d} \\
\rho=M \ddot{q}_{d}+C_{0} \dot{q}_{d}+G(q)+F(\dot{q})
\end{array}\right. \text {. }
$$

It is assumed that the control gain matrices are positive definite. 
Theorem 1. For an n-DOF serial robotic manipulator described in Equation (1), if the proposed CC-PD control law in Equation (9) is applied to the system, the controlled robotic manipulator is globally stable for contouring control with bounded tracking errors, provided that the following conditions in Equation (15) are satisfied.

$$
\left\{\begin{array}{l}
\bar{K}_{d} \succ 0, \quad \bar{K}_{p} \succ M \succ 0 \\
K_{p p} \succ 0, \quad K_{d d} \succ 0 \\
K_{d d}+C_{0}-M \succ 0 \\
\bar{K}_{p}-K_{p p}+\bar{K}_{d}-K_{d d}+C^{T}-C_{0} \succ 0 \\
\lambda_{m}\left(K_{d d}+C_{0}-M\right)>\frac{1}{2} \lambda_{M}\left(\bar{K}_{p}-K_{p p}+\bar{K}_{d}-K_{d d}+C^{T}-C_{0}\right)>0 \\
\lambda_{m}\left(K_{p p}\right)>\frac{1}{2} \lambda_{M}\left(\bar{K}_{p}-K_{p p}+\bar{K}_{d}-K_{d d}+C^{T}-C_{0}\right)>0
\end{array}\right.
$$

where $\bar{K}_{p}$ and $\bar{K}_{d}$ are user-defined symmetric positive definite matrices.

\section{Proof of Stability of CC-PD Controller}

To prove the stability of the proposed CC-PD contouring controller, the Lyapunov theorem $[20,22]$ is used by defining the following Lyapunov function candidate as:

$$
V=\frac{1}{2}\left[\begin{array}{ll}
e_{q} & \dot{e}_{q}
\end{array}\right]^{T} L\left[\begin{array}{l}
e_{q} \\
\dot{e}_{q}
\end{array}\right]
$$

where $L=\left[\begin{array}{cc}\bar{K}_{p}+\bar{K}_{d} & M \\ M & M\end{array}\right]$.

As matrices $\bar{K}_{p}, \bar{K}_{d}$, and $M$ are symmetric positive definite, then matrix $L$ is also symmetric. From condition Equation (15) and property P5, it can be easily proven that:

$$
\left\{\begin{array}{l}
M^{-1} \succ \bar{K}_{p}^{-1} \succ 0 \\
M^{-1}-\bar{K}_{p}^{-1} \succ 0
\end{array}\right.
$$

Applying property P6 to Equation (17), we have:

$$
S=M-M \bar{K}_{p}^{-1} M \succ 0
$$

From Equation (18), it can be seen that the Schur complement [21] $S$ of matrix $\bar{K}_{p}$ in matrix $L$ is symmetric positive definite. According to the proposition introduced in [20,22], it is demonstrated that matrix $L$ is symmetric positive definite. In addition, the control gain matrix $\bar{K}_{d}$ is symmetric positive definite. Therefore, the Lyapunov function $V$ in Equation (16) is a positive definite function, i.e., $V \geq 0$.

Differentiating Equation (16) with respect to time, we have:

$$
\dot{V}=\left[\begin{array}{cc}
e_{q} & \dot{e}_{q}
\end{array}\right]^{T}\left[\begin{array}{cc}
\bar{K}_{p}+\bar{K}_{d} & M \\
M & M
\end{array}\right]\left[\begin{array}{c}
\dot{e}_{q} \\
\ddot{e}_{q}
\end{array}\right]+\frac{1}{2}\left[\begin{array}{ll}
e_{q} & \dot{e}_{q}
\end{array}\right]^{T}\left[\begin{array}{cc}
0 & \dot{M} \\
\dot{M} & \dot{M}
\end{array}\right]\left[\begin{array}{c}
e_{q} \\
\dot{e}_{q}
\end{array}\right]
$$

Substituting $M \ddot{e}_{q}$ from Equation (14) into Equation (19) and using properties P2 and P3 yields

$$
\dot{V}=-\dot{e}_{q}^{T}\left(K_{d d}+C_{0}-M\right) \dot{e}_{q}-e_{q}^{T} K_{p p} e_{q}+e_{q}^{T}\left(\bar{K}_{p}-K_{p p}+\bar{K}_{d}-K_{d d}+C^{T}-C_{0}\right) \dot{e}_{q}+\left(e_{q}+\dot{e}_{q}\right)^{T} \rho
$$

From Equation (15), it is easy to prove that

$$
e_{q}^{T}\left(\bar{K}_{p}-K_{p p}+\bar{K}_{d}-K_{d d}+C^{T}-C_{0}\right) \dot{e}_{q} \leq \frac{\lambda_{M}\left(\bar{K}_{p}-K_{p p}+\bar{K}_{d}-K_{d d}+C^{T}-C_{0}\right)}{2}\left(e_{q}^{T} e_{q}+\dot{e}_{q}^{T} \dot{e}_{q}\right)
$$

From property $\mathrm{P} 4$, it can be proven that parameter $\rho$ is bounded with $\rho_{b}(>0)$ as the upper bound. It means $\rho \leq\|M\|\left\|\ddot{q}_{d}\right\|+\left\|C_{0}\right\|\left\|\dot{q}_{d}\right\|+\|G(q)\|+\|F(q, \dot{q})\|=\rho_{b}$. 
Therefore, the following inequality is hold:

$$
\left(e_{q}+\dot{e}_{q}\right)^{T} \rho \leq\left(\left\|e_{q}\right\|+\left\|\dot{e}_{q}\right\|\right) \rho_{b}
$$

Substituting Equations (21) and (22) into Equation (20) yields

$$
\begin{aligned}
\dot{V} & =-\dot{e}_{q}^{T}\left(K_{d d}+C_{0}-M-\frac{\lambda_{M}\left(\bar{K}_{p}-K_{p p}+\bar{K}_{d}-K_{d d}+C^{T}-C_{0}\right)}{2} I\right) \dot{e}_{q} \\
& -e_{q}^{T}\left(K_{p p}-\frac{\lambda_{M}\left(\bar{K}_{p}-K_{p p}+\bar{K}_{d}-K_{d d}+C^{T}-C_{0}\right)}{2}\right) e_{q}+\left(\left\|e_{q}\right\|+\left\|\dot{e}_{q}\right\|\right) \rho_{b}
\end{aligned}
$$

According to Equation (15), it can be seen that

$$
\left\{\begin{array}{l}
\gamma_{e}=\lambda_{m}\left(K_{p p}-\frac{\lambda_{M}\left(\bar{K}_{p}-K_{p p}+\bar{K}_{d}-K_{d d}+C^{T}-C_{0}\right)}{2} I\right)>0 \\
\gamma_{\dot{e}}=\lambda_{m}\left(K_{d d}+C_{0}-M-\frac{\lambda_{M}\left(\bar{K}_{p}-K_{p p}+\bar{K}_{d}-K_{d d}+C^{T}-C_{0}\right)}{2} I\right)>0
\end{array}\right.
$$

Applying Equation (24) into Equation (23) yields

$$
\dot{V} \leq-\gamma_{\dot{e}}\left\|\dot{e}_{q}\right\|^{2}+\rho_{b}\left\|\dot{e}_{q}\right\|-\gamma_{e}\left\|e_{q}\right\|^{2}+\rho_{b}\left\|e_{q}\right\|
$$

For all positive parameters, the following inequalities hold:

$$
\left\{\begin{array}{l}
-\gamma_{e}\left\|e_{q}\right\|^{2}+\rho_{b}\left\|e_{q}\right\| \leq-\frac{\gamma_{e}}{4}\left\|e_{q}\right\|^{2}+\frac{\left(\rho_{b}\right)^{2}}{\gamma_{e}} \\
-\gamma_{\dot{e}}\left\|\dot{e}_{q}\right\|^{2}+\rho_{b}\left\|\dot{e}_{q}\right\| \leq-\frac{\gamma_{\dot{e}}}{4}\left\|\dot{e}_{q}\right\|^{2}+\frac{\left(\rho_{b}\right)^{2}}{\gamma_{\dot{e}}}
\end{array}\right.
$$

Substituting Equation (26) into Equation (25) yields

$$
\dot{V} \leq-\frac{\gamma_{\dot{e}}}{4}\left\|\dot{e}_{q}\right\|^{2}-\frac{\gamma_{e}}{4}\left\|e_{q}\right\|^{2}+\left(\frac{\gamma_{e}+\gamma_{\dot{e}}}{\gamma_{e} \gamma_{\dot{e}}}\right)\left(\rho_{b}\right)^{2}
$$

From Equation (27), it immediately follows that

$$
\dot{V}<0, \forall\left\{\begin{array}{l}
\left\|e_{q}\right\|>\delta_{e}=\frac{2 \rho_{b}}{\gamma_{e}} \sqrt{\left(\frac{\gamma_{e}+\gamma_{\dot{e}}}{\gamma_{\dot{e}}}\right)} \\
\left\|\dot{e}_{q}\right\|>\delta_{\dot{e}}=\frac{2 \rho_{b}}{\gamma_{\dot{e}}} \sqrt{\left(\frac{\gamma_{e}+\gamma_{\dot{e}}}{\gamma_{e}}\right)}
\end{array}\right.
$$

In other words, the time derivative of the Lyapunov function $V$ is negative outside the compact set $B_{\delta}=\left\{\left\|e_{q}\right\| \leq \delta_{e},\left\|\dot{e}_{q}\right\| \leq \delta_{\dot{e}}\right\}$, or equivalently, all solutions that start outside of $B_{\delta}$ will re-enter the compact set $B_{\delta}$ within a finite time, and will remain there afterward. According to the Lyapunov theorem [22], the controlled robotic manipulator is globally uniformly bounded, and the bounded tracking errors can be obtained as follows:

$$
\left\{\begin{aligned}
&\left\|e_{q}\right\| \leq \frac{2 \rho_{b}}{\gamma_{e}} \sqrt{\left(\frac{\gamma_{e}+\gamma_{\dot{e}}}{\gamma_{\dot{e}}}\right)} \\
&\left\|\dot{e}_{q}\right\| \leq \frac{2 \rho_{b}}{\gamma_{\dot{e}}} \sqrt{\left(\frac{\gamma_{e}+\gamma_{\dot{e}}}{\gamma_{e}}\right)}
\end{aligned}\right.
$$

From Equation (28), it can be seen that the tracking errors are controlled by the proportional and derivative gains. Generally speaking, a large control gain $K_{p p}$ (that is the combination of proportional gains in the joint space and the task space) will increase the value of $\gamma_{e}$, while a large control gain $K_{d d}$ (that is the combination of derivative gains in these two spaces) will increase the value of $\gamma_{\dot{e}}$. 
From Equation (28), it is concluded that increasing both control gains will reduce the tracking errors. However, the control gains cannot be chosen too large concerning the vibration of the system caused by the amplified noises and disturbances. Therefore, a try-and-error method is used to determine the control gains in this paper.

\section{Simulation Verification and Discussion}

In this section, some simulation study results are presented to demonstrate the effectiveness of the proposed CC-PD controller. A 3-DOF serial robotic manipulator shown in Figure 2, with structural parameters listed in Table 1, is used for contour tracking control experiments. The configuration of joints in this paper is in the vertical direction, and the dynamic model can be found in [23,24].

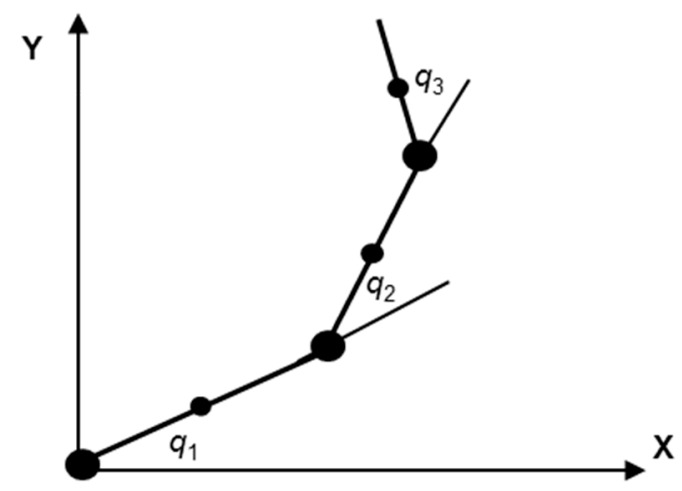

Figure 2. Scheme of a 3-DOF robotic manipulator.

Table 1. Structural parameters of a 3-DOF robotic manipulator.

\begin{tabular}{ccccc}
\hline Link $\boldsymbol{i}$ & Mass $\boldsymbol{m}_{\boldsymbol{i}} \mathbf{( \mathbf { k g } )}$ & Length $\boldsymbol{l}_{\boldsymbol{i}}(\mathbf{m})$ & Mass Center $\boldsymbol{r}_{\boldsymbol{i}}(\mathbf{m})$ & Inertia $\left.\boldsymbol{I}_{\boldsymbol{i}} \mathbf{( k g} \cdot \mathbf{m}^{\mathbf{2}}\right)$ \\
\hline 1 & 1.0 & 0.50 & 0.25 & 0.10 \\
2 & 1.0 & 0.50 & 0.25 & 0.10 \\
3 & 0.5 & 0.30 & 0.15 & 0.05 \\
\hline
\end{tabular}

In the simulation study, the following friction model in the robot joints [25] is used:

$$
F\left(\dot{q}_{i}\right)=\left[F_{c}+F_{s} e^{-\left|\dot{q}_{i}\right|_{s}^{\alpha}}\right] \operatorname{sign}\left(\dot{q}_{i}\right)+F_{v} \dot{q}_{i}
$$

where the friction parameters are selected as: $F_{c}=3.4 \times 10^{-2}, F_{s}=4.5 \times 10^{-2}, F_{v}=3.7 \times 10^{-4}$, $\dot{q}_{s}=10.7$, and $\alpha=2$.

\subsection{Linear Motion Contour Tracking}

In the first example, a linear motion of the end-effector, which is defined from point $A(0.5,0.0)$ to point $B(0.2,1.0)$ with a constant orientation of $\pi / 3$ for time duration of $4 \mathrm{~s}$, is tracked for the 3-DOF robotic manipulator. To form a smooth motion in each joint, the 5 th order polynomial trajectories for positions at the end-effector level are designed, and the inverse kinematics analysis is conducted to get positions and velocities of the joints [20].

In all simulations for linear contour motions, the following control gains are selected based on Equation (15) and through the trial-and-error method.

$$
\left\{\begin{array} { l } 
{ K _ { p } = \operatorname { d i a g } ( 3 0 0 0 , 3 0 0 0 , 3 0 0 0 ) } \\
{ K _ { d } = \operatorname { d i a g } ( 2 0 0 0 , 2 0 0 0 , 2 0 0 0 ) }
\end{array} \text { and } \left\{\begin{array}{l}
K_{c p}=[750,750,750]^{T} \\
K_{c d}=[500,500,500]^{T}
\end{array}\right.\right.
$$


Figure 3 shows the simulation results for tracking errors, controlled torques, and tracking errors and contour errors in the end-effector under PD control and CC-PD control, respectively. From Figure 3, one can see that the overall trajectory tracking and contour tracking performances in the task space, as shown in Figure 3c, are better controlled by the proposed CC-PD control than those controlled by the PD control, although the required control torques under these two control laws are very similar, see Figure $3 b$, and the overall tracking errors in the joint space under PD control are slightly smaller than those under CC-PD control, especially for joints 2 and 3, see Figure 3a. It demonstrates the effectiveness of the cross-coupled control action in the CC-PD control law for contour tracking performance improvement at the end-effector level.
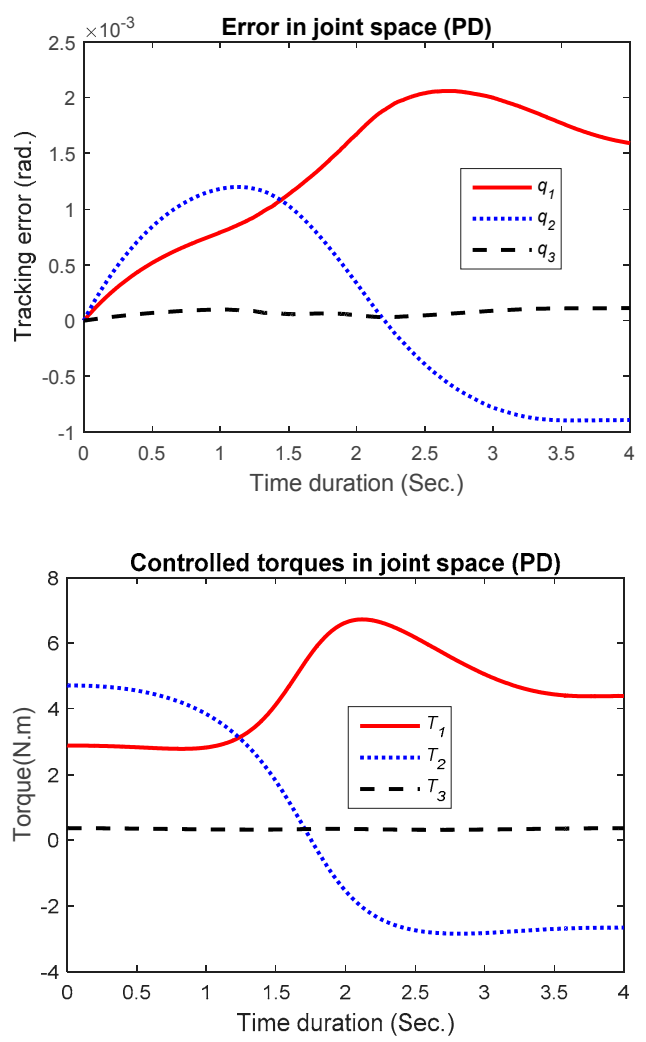

(a)
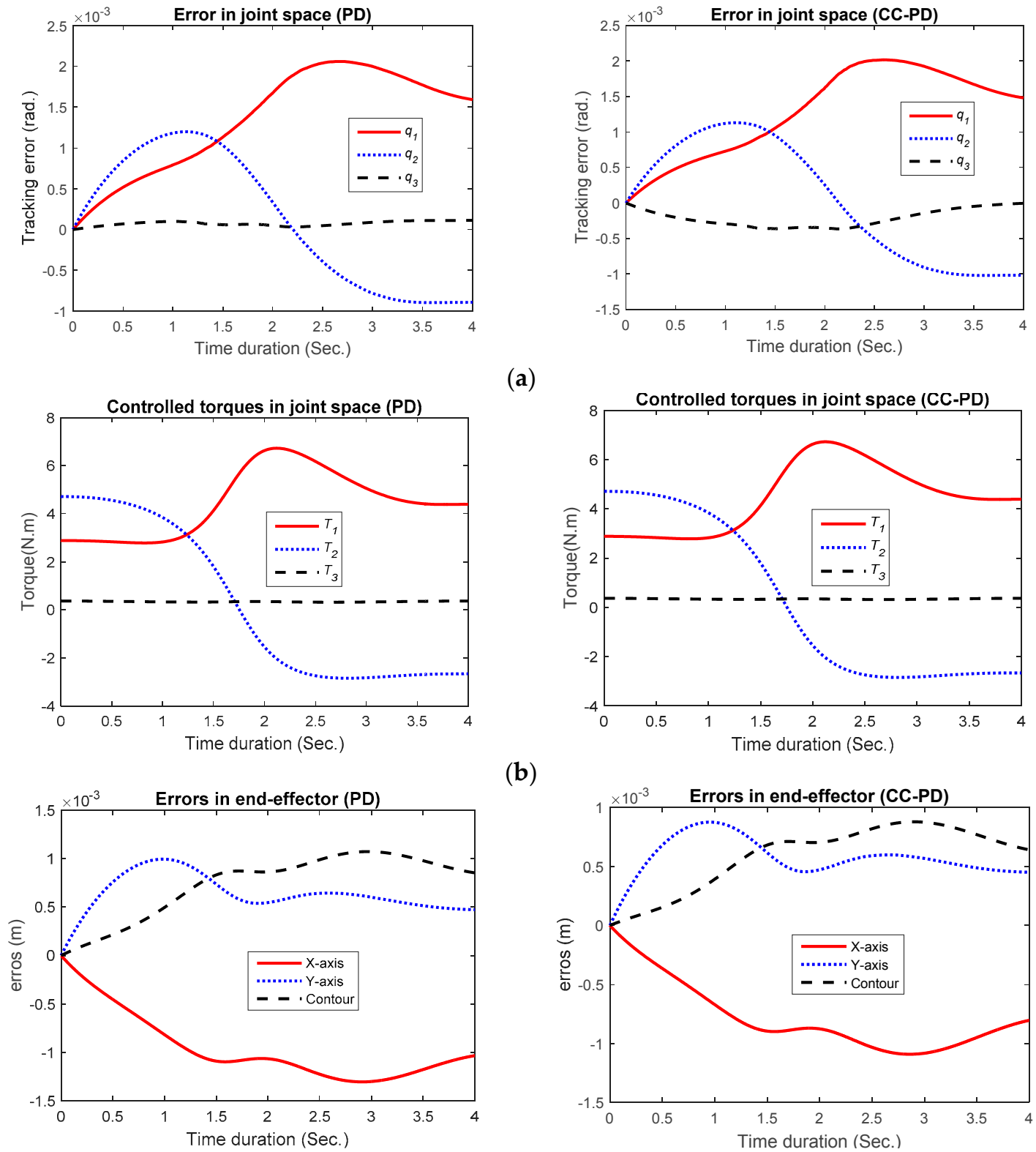

(b)

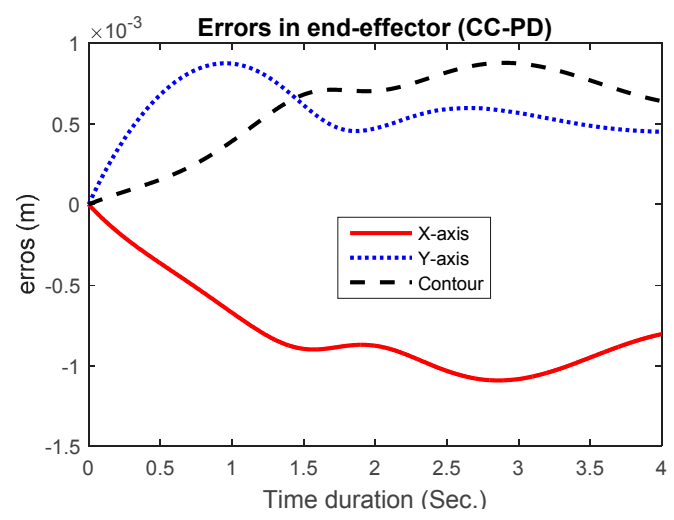

(c)

Figure 3. Tracking performance comparison under PD and CC-PD controllers. (a) Tracking errors for PD control and CC-PD control; (b) Controlled torques for PD control and CC-PD control; (c) Tracking and contour errors of the end-effector for PD control and CC-PD control. 
To exam the effect of the cross-coupled gains $\left(K_{c p}\right.$ and $\left.K_{c d}\right)$ on contour tracking performance improvement, different factors for CC control gains are selected and simulations are conducted. Figure 4 shows the contour tracking results for different cross-coupled gains for the factors from 0 to $4(\times 0$ to $\times 4)$, where CC-PD $(\times 0)$ represents the PD control, and $\times 4$ means the CC control gains are increased to four times of the original CC gains in Equation (30). From this figure, it is clearly shown that the improvement trends of contour tracking performances with the increase of the CC control gains. This result is coincident with the theorem and the analysis conducted in the previous section.

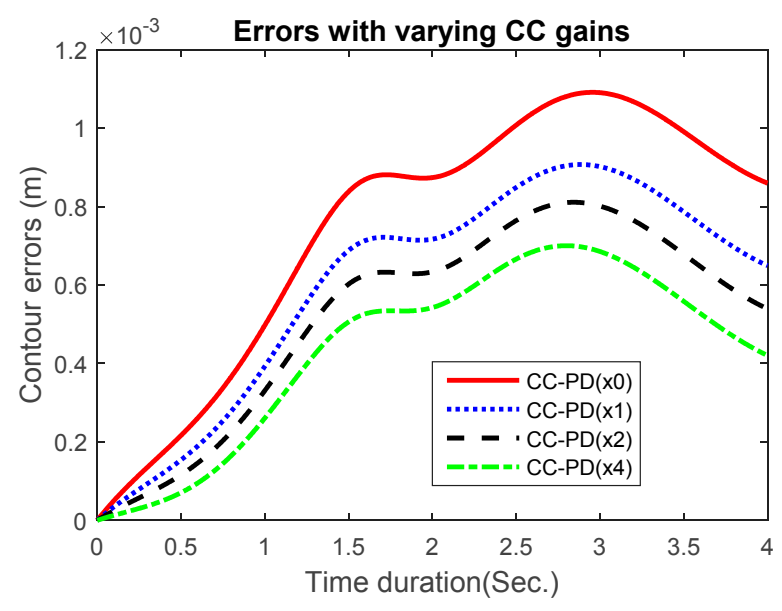

Figure 4. Contour tracking performance improvement for linear motion under different CC gains.

The second example is the contour tracking of a vertical line in the end-effector level. The vertical line is defined from $C(0.5,0)$ to $\mathrm{D}(0.5,0.6)$ with a varying orientation from 0 to $\mathrm{pi} / 4$, and is tracked under the same control gains in Equation (30). Figure 5 shows the simulation results for the tracking errors in the joint level and the end-effector level under PD and CC-PD control, respectively. Compared the tracking error results for PD and CC-PD control shown in Figure 5b, one can see that the CC-PD control also obtained a better contour tracking performance (black dashed lines) in the end-effector, even compatible tracking errors were obtained in the joint level for both PD and CC-PD control, shown in Figure 5a. From this example and the previous example, one can see the suitability of the same control gains for different tracking problems.

Figure 6 shows the contour tracking performance improvement at the end-effector level for the vertical line under different CC control gains. Once again, it demonstrates that the larger the CC control gains, the smaller the contour errors. The maximum contour error is about $1.3 \times 10^{-3} \mathrm{~m}$ when only the PD control is used (CC-PD $(\times 0))$, while the maximum contour errors are about $0.95 \times 10^{-3} \mathrm{~m}$, $0.75 \times 10^{-3} \mathrm{~m}$, and $0.55 \times 10^{-3} \mathrm{~m}$ for CC-PD control with original CC gains, doubled CC gains, and quadrupled CC gains, respectively. 

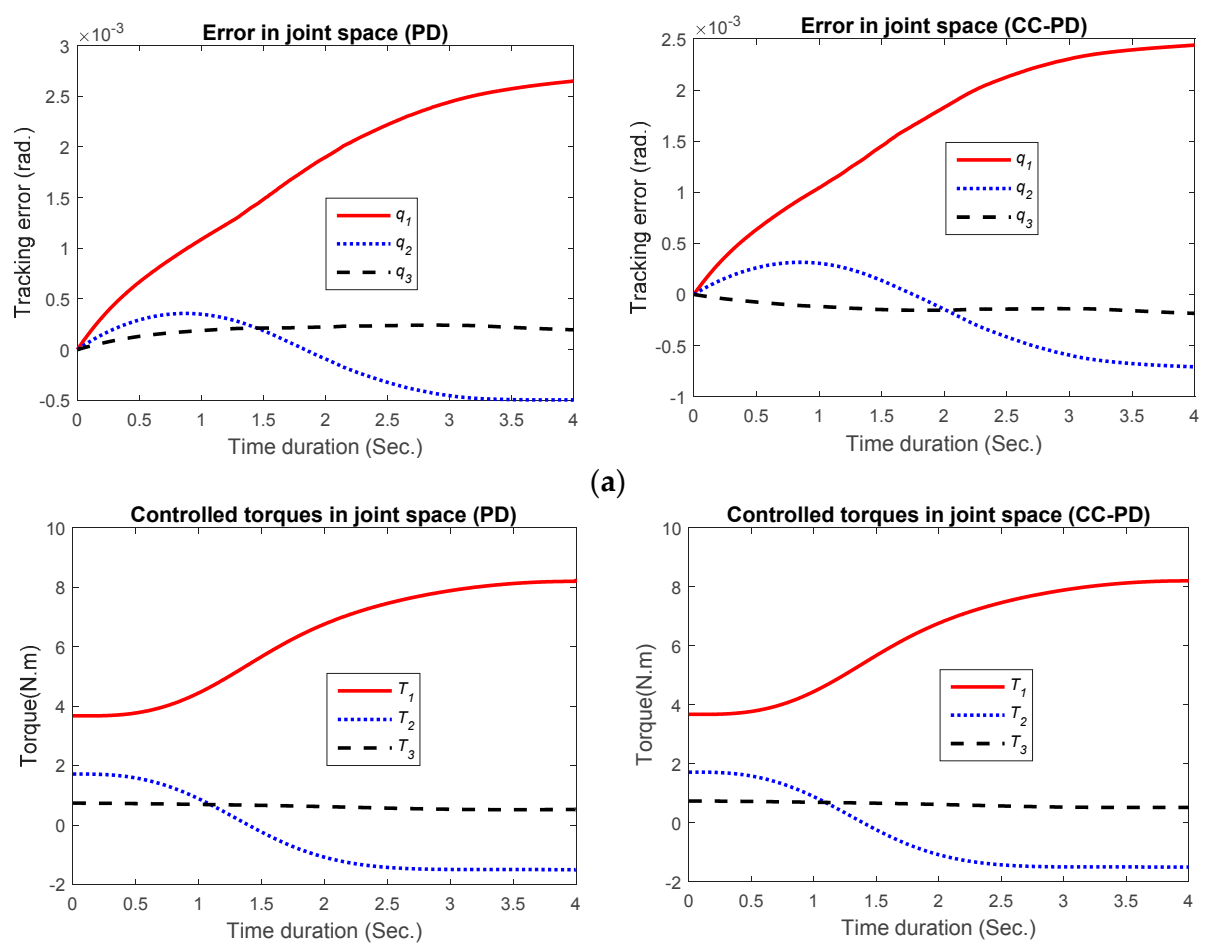

(a)

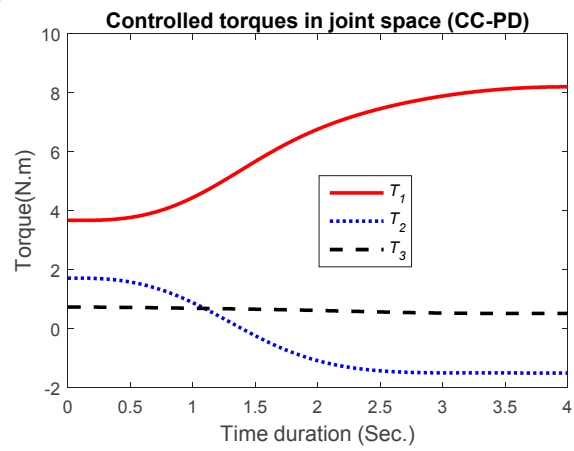

(b)
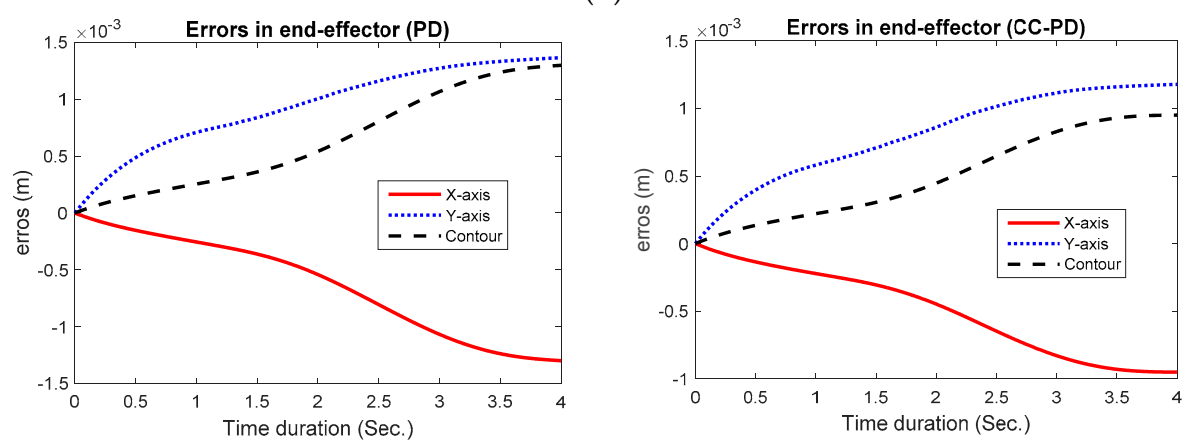

(c)

Figure 5. Tracking control results for a vertical line under PD and CC-PD control; (a) Tracking errors in joint space for PD control and CC-PD control; (b) Controlled torques for PD control and CC-PD control; (c) Tracking and contour errors of the end-effector for PD control and CC-PD control.

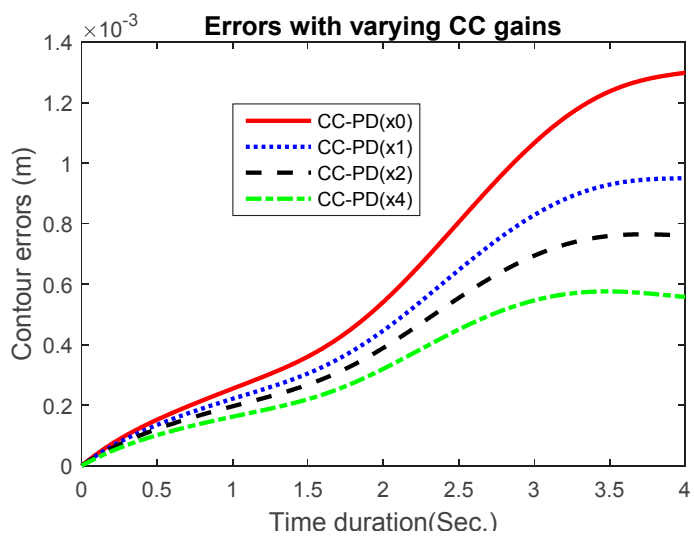

Figure 6. Comparison of contour errors for vertical line tracking under different CC gains. 


\subsection{Circular Contour Tracking}

Circular contour tracking control is more complicated than the linear contour tracking because of the nonlinear property of the contour error coefficients in Equation (4). In this simulation, a circular contour with a radius of $0.6 \mathrm{~m}$ and the center at $(0,0)$ is tracked in $10 \mathrm{~s}$, using the same control gains listed in Equation (30).

Figure 7 presents the comparison results based on PD and CC-PD control laws. Figure 7a shows the tracking errors in the joint space, Figure $7 \mathrm{~b}$ is the corresponding controlled torques, and Figure $7 \mathrm{c}$ is the tracking and contour errors at the end-effector under PD and CC-PD control, respectively. The maximum contour errors are $8.8 \times 10^{-4} \mathrm{~m}$ under PD control and $7.0 \times 10^{-4} \mathrm{~m}$ under CC-PD control. It is shown that, under very similar controlled torques shown in Figure $7 \mathrm{~b}$, the CC-PD control obtained better contour tracking performance than the PD control, even in a degraded tracking performance for joint 3 compared with the counterpart of the PD control, as shown in Figure 7a. Therefore, the effectiveness of the proposed CC-PD control is verified for complex contour tracking problems.
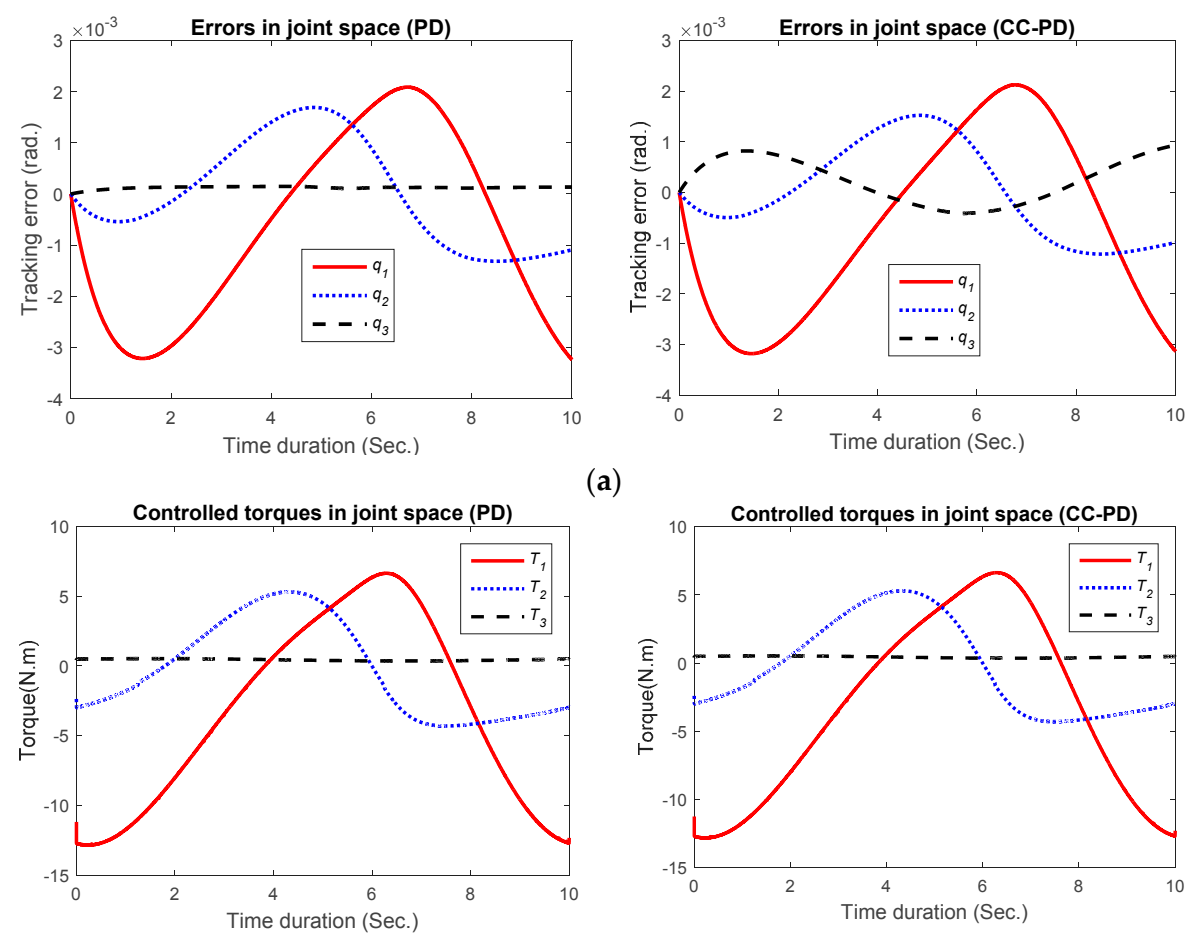

(a)

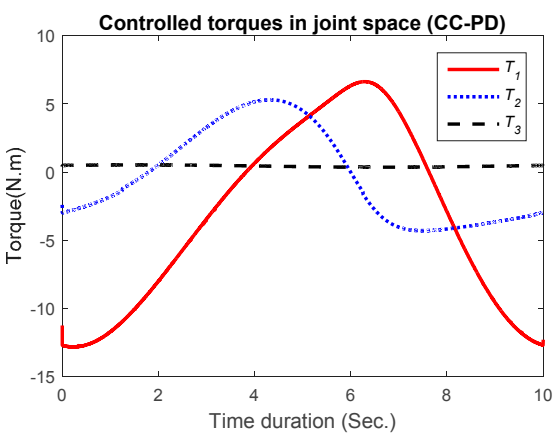

(b)
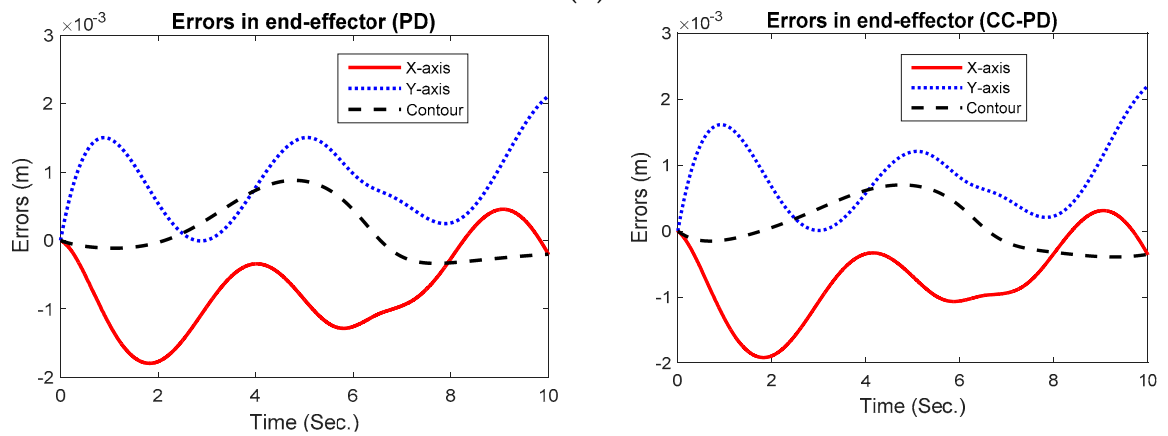

(c)

Figure 7. Comparison of tracking errors for circular contour under PD and CC-PD; (a) Tracking errors in joint space for PD control and CC-PD control; (b) Controlled torques for PD control and CC-PD control; (c) Tracking and contour errors of the end-effector for PD control and CC-PD control. 
To test the effort of the CC control gains on the contour tracking performance improvement for circular motions, different factors are used with varying $\mathrm{CC}$ gains and the contour tracking results are shown in Figure 8 where the red curve CC-PD $(\times 0)$ represents the PD control contour error. It also shows that, with the increase of the $\mathrm{CC}$ control gains, the maximum contour errors become smaller and smaller, resulting in a similar conclusion obtained in linear contour tracking control.

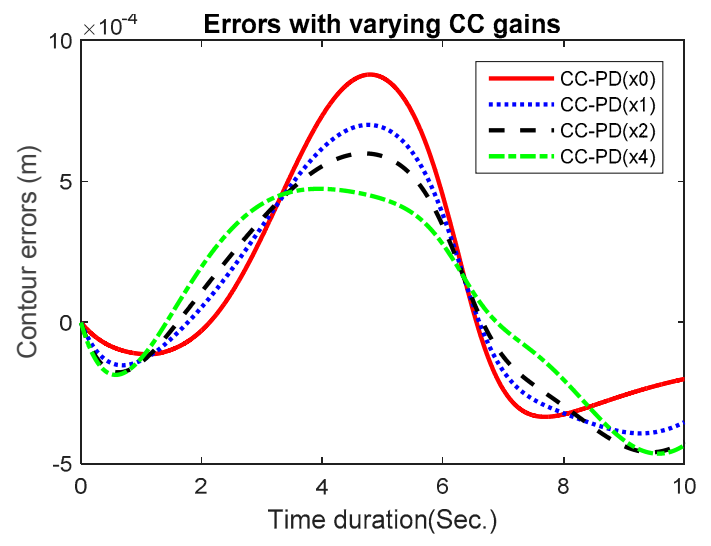

Figure 8. Contour tracking performance improvement with the increase of CC control gains.

To verify the effect of the control gains on the contour tracking performance improvement, high control gains with a factor of 10 are used for both PD and CC-PD control in the circular contour tracking example, and Figure 9 shows the contour errors for both control laws. Compared with Figure 8, one can see that the contour errors are reduced around 10 times when the control gains are increased 10 times. It demonstrates that the control gains have significant contributions for contouring control, and it proves the theorem developed in this paper.

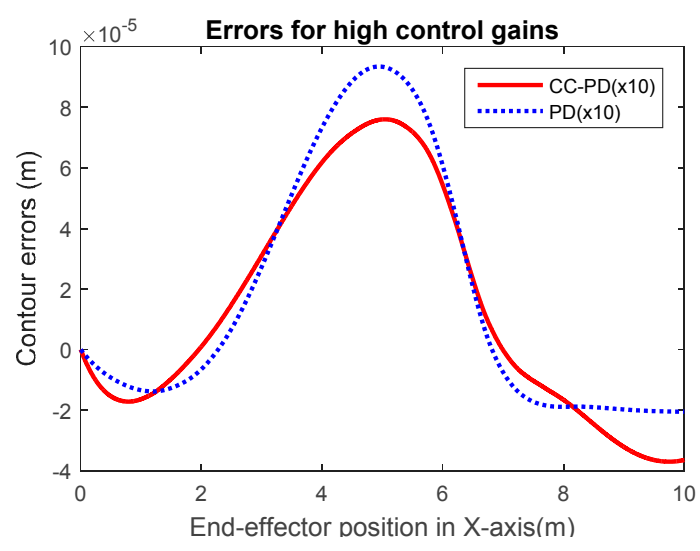

Figure 9. Contour errors under high control gains for circular contour tracking.

\subsection{Discussions}

In all the simulation studies, the same control gains were used for contour tracking of both linear and nonlinear contours; good tracking performances were obtained for PD and CC-PD control, and better contour tracking performances were obtained by CC-PD control. Such results demonstrated the effectiveness of the CC-PD control and the simplicity of choosing control gains in the implementation. In addition, simulation results also proved that large control gains will reduce the contour errors that are coincident with the theorem proposed in this paper. Of course, in order to obtain good tracking performances for different contours, tuning the control gains properly will be a good practice. 
It should be mentioned that the same PD control gains were used for both PD and CC-PD control in the simulations, which is a simplification process for the purpose of comparison study. In order to pursuit a comprehensive and fair comparison between PD control and CC-PD control, it is necessary to use some artificial intelligent algorithms such as GA, PSO, and DE $[26,27]$ to optimize the control gains based on contour tracking performances and the bounded control torques, and that will be a desired future research work.

In this paper, only the linear and circular contours were tracked under the PD and CC-PD control laws. Theoretically, if the contour error estimation methods in $[28,29]$ are used to replace Equation (4), the proposed CC-PD control can be applied to free-path contour tracking problems, and that will be a future research work. Additionally, this proposed control method can be applied in position domain $[30,31]$ for contour tracking performance improvement.

\section{Conclusions}

Contour tracking control is one of the most important tasks for motion control of the end-effector of a robotic manipulator. In this paper, a cross-coupled contouring control called CC-PD control was proposed for linear and nonlinear contour tracking control to a multi-DOF robotic manipulator. The proposed CC-PD control consists of a PD control in joint level for system stability and a PD contour control at the end-effector for contour tracking performance improvement. Based on the Jacobian regulation, the contour error was mapped to the joint tracking errors and the CC-PD control is implemented at the joint level.

Stability analysis was conducted for the proposed CC-PD control and simulation verification study was performed. It is demonstrated that the CC-PD control is better than the PD control in terms of the reduction of contour errors at the end-effector level for linear and nonlinear contour cases. It also shows that the contour tracking performance can be improved with the increase of cross-coupled control gains. More complicated contour tracking control and the optimization of control gains for the proposed CC-PD control are some of the future work.

Acknowledgments: This research is supported by the Natural Sciences and Engineering Research Council of Canada (NSERC) through a Discovery Grant, and the Hunan Province innovation platform and talent plan (Applied Fundamental Research) No. 2015JC3109.

Author Contributions: P.O. and D.L. conceived and designed the direction of research; P.O. and Y.H. derived the main theorem and proof; Y.H. and W.Y. conducted the simulation study; D.L. advised and revised the final contents in the paper; all authors contributed in finishing this paper, and all authors have read and approved the final manuscript.

Conflicts of Interest: The authors declare no conflict of interest.

\section{References}

1. Koren, Y. Cross-coupled biaxial computer controls for manufacturing systems. ASME J. Dyn. Syst. Meas. Control 1980, 102, 265-272. [CrossRef]

2. Koren, Y.; Lo, C.C. Variable-gain cross-coupling controller for contouring. CIRP Ann. Manuf. Technol. 1991, 40, 371-374. [CrossRef]

3. Shih, Y.Y.; Chen, C.S.; Lee, A.C. A novel cross-coupling control design for bi-axis motion. Int. J. Mach. Tools Manuf. 2002, 42, 1539-1548. [CrossRef]

4. Yang, J.Z.; Li, Z.X. A novel contour error estimation for position loop-based cross-coupled control. IEEE/ASME Trans. Mechatron. 2011, 16, 643-655. [CrossRef]

5. Cheng, M.Y.; Su, K.H.; Wang, S.F. Contour error reduction for free-form contour following tasks of biaxial motion control systems. Robot. Comput. Integr. Manuf. 2009, 25, 323-333. [CrossRef]

6. Ramesh, R.; Mannan, M.A.; Poo, A.N. Tracking and contour error control in CNC servo systems. Int. J. Mach. Tools Manuf. 2005, 45, 301-326. [CrossRef]

7. Tang, L.; Landes, R.G. Multiaxis contour control-The state of the art. IEEE Trans. Control Syst. Technol. 2013, 21, 1997-2010. [CrossRef] 
8. El Khalick, A.; Uchiyama, M.N. Contouring controller design based on iterative contour error estimation for three-dimensional machining. Robot. Comput. Integr. Manuf. 2011, 27, 802-807. [CrossRef]

9. Chen, C.S.; Chen, L.Y. Cross-coupling position command control in a multi-axis motion system. Mechatronics 2011, 21, 625-632. [CrossRef]

10. Cheah, C.C. Task-space PD control of robot manipulators: Unified analysis and duality property. Int. J. Robot. Res. 2008, 27, 1152-1170. [CrossRef]

11. Cheah, C.C.; Liu, C.; Slotine, J.J.E. Adaptive tracking control for robots with unknown kinematic and dynamic properties. Int. J. Robot. Res. 2006, 25, 283-296. [CrossRef]

12. Chen, S.; Wu, K. Contouring control of smooth paths for multiaxis motion systems based on equivalent errors. IEEE Trans. Control Syst. Technol. 2007, 1, 1151-1158. [CrossRef]

13. Chen, S.L.; Li, H.L.; Ting, S.C. Contouring control of biaxial systems based on polar coordinates. IEEE/ASME Trans. Mechatron. 2002, 7, 329-345. [CrossRef]

14. Yao, B.; Hu, C.X.; Wang, Q.F. An orthogonal global task coordinate frame for contouring control of biaxial systems. IEEE/ASME Trans. Mechatron. 2012, 17, 622-634.

15. Lee, J.H.; Dixon, W.E.; Ziegert, J.C. Adaptive nonlinear contour coupling control for a machine tool system. Int. J. Adv. Manuf. Technol. 2012, 61, 1057-1065. [CrossRef]

16. Fang, R.W.; Chen, J.S. A cross-coupling controller using an $\mathrm{H}_{\infty}$ scheme and its application to a two-axis direct-drive robot. J. Robot. Syst. 2002, 19, 483-497. [CrossRef]

17. Chen, C.L.; Wu, T.C.; Peng, C.C. Robust trajectories following control of a 2-link robot manipulator via coordinate transformation for manufacturing applications. Robot. Comput. Integr. Manuf. 2011, 27, 569-580. [CrossRef]

18. Fang, R.W.; Chen, J.S. Cross-coupling control for a direct-drive robot. JSME Int. J. Ser. C Mech. Syst. Mach. Elem. Manuf. 2002, 45, 749-757. [CrossRef]

19. Ouyang, P.R.; Hu, Y.Q.; Yue, W.H.; Liu, D.S. Cross coupling contour tracking of multi-DOF robot. In Proceedings of the 2014 IEEE International Conference on Robotics and Biomimetics, Bali, Indonesia, 5-10 December 2014; pp. 1463-1468.

20. Craig, J.J. Introduction to Robotics: Mechanics and Control; Addison-Wesley: Reading, MA, USA, 1986.

21. Arendt, W.; Schleich, W.P. Mathematical Analysis of Evolution, Information, and Complexity; Wiley-VCH: Weinheim, Germany, 2009.

22. Slotine, J.J.E.; Li, W.P. Applied Nonlinear Control; Prentice-Hall, Inc.: Upper Saddle River, NJ, USA, 1991.

23. Ouyang, P.R.; Pano, V.; Acob, J. Position domain synchronization control of multi-degrees of freedom of robotic manipulator. Trans. ASME J. Dyn. Syst. Meas. Control 2014, 136. [CrossRef]

24. Lynch, K.M.; Shiroma, N.; Arai, H.; Tanie, K. Collision-free trajectory planning for a 3-DOF robot with a passive joint. Int. J. Robot. Res. 2000, 19, 1171-1184. [CrossRef]

25. Bittencourt, A.C.; Gunnarson, S. Static friction in a robot joint-Modeling and identification of load and temperature effects. Trans. ASME J. Dyn. Syst. Meas. Control 2012, 134. [CrossRef]

26. Hassan, R.; Cohanim, B.; De Weck, O.; Venter, G. A comparison of particle swarm optimization and the genetic algorithm. AIAA/ASME/ASCE/AHS/ASC Struct. Struct. Dyn. Mater. Conf. 2005, 2, 1138-1150.

27. Ouyang, P.R.; Pano, V. Comparative study of DE, PSO and GA for position domain PID controller tuning. Algorithms 2015, 8, 697-711. [CrossRef]

28. Cheng, M.Y.; Lee, C.C. Motion controller design for contour-following tasks based on real-time contour error estimation. IEEE Trans. Ind. Electron. 2007, 54, 1686-1695. [CrossRef]

29. Wu, J.H.; Xiong, Z.H.; Ding, H. Integral design of contour error model and control for biaxial system. Int. J. Mach. Tools Manuf. 2015, 89, 159-169. [CrossRef]

30. Ouyang, P.R.; Pano, V.; Acob, J. Position domain contour control for multi-dof robotic system. Mechatron. 2013, 23, 1061-1071. [CrossRef]

31. Ouyang, P.R.; Pano, V.; Dam, T. PID position domain control for contour tracking. Int. J. Syst. Sci. 2015, 46, 111-124. [CrossRef]

(C) 2016 by the authors; licensee MDPI, Basel, Switzerland. This article is an open access article distributed under the terms and conditions of the Creative Commons Attribution (CC-BY) license (http:/ / creativecommons.org/licenses/by/4.0/). 